\title{
Oligodendrocyte Bioenergetics in Health and Disease
}

\author{
Lauren Rosko',2, Victoria N. Smith', Reiji Yamazaki', \\ and Jeffrey K. Huang ${ }^{1,2,3}$ (ic
}

\begin{abstract}
The human brain weighs approximately $2 \%$ of the body; however, it consumes about $20 \%$ of a person's total energy intake. Cellular bioenergetics in the central nervous system involves a delicate balance between biochemical processes engaged in energy conversion and those responsible for respiration. Neurons have high energy demands, which rely on metabolic coupling with glia, such as with oligodendrocytes and astrocytes. It has been well established that astrocytes recycle and transport glutamine to neurons to make the essential neurotransmitters, glutamate and GABA, as well as shuttle lactate to support energy synthesis in neurons. However, the metabolic role of oligodendrocytes in the central nervous system is less clear. In this review, we discuss the energetic demands of oligodendrocytes in their survival and maturation, the impact of altered oligodendrocyte energetics on disease pathology, and the role of energetic metabolites, taurine, creatine, $\mathrm{N}$-acetylaspartate, and biotin, in regulating oligodendrocyte function.
\end{abstract}

\section{Keywords}

oligodendrocytes, bioenergetics, multiple sclerosis, remyelination, neurodegeneration

\section{Energetic Demands in Oligodendrocytes}

During peak myelination, an oligodendrocyte expands its membrane three times its weight per day and eventually supports a membrane weight of 100 times its cell body (Bradl and Lassmann 2010; McLaurin and Yong 1995). Oligodendrocytes consume large amounts of metabolites, such as glucose and lactate, for the energy-demanding tasks of myelination during development and remyelination after demyelinating injury (Fig. 1). The significant energy and metabolic substrate demands for myelination are supplied from the diet or from reserves of protein, fat, and glycogen that must later be replaced. It is estimated that $3.3 \times 10^{23}$ ATP molecules are needed for oligodendrocytes to synthesize just one gram of myelin (Harris and Attwell 2012). Despite the large initial energy demands necessary for myelin synthesis, mature myelin is energetically favorable because it reduces the energy demands required for action potentials and accelerates nerve conduction. In addition to their role in myelination, oligodendrocytes facilitate the transfer of metabolites to fuel neurons and support the integrity of myelinated axons (Griffiths and others 1998). Axons have high energy costs since they must maintain $\mathrm{Na}^{+} / \mathrm{K}^{+}$-ATPase pumps and restore ionic gradients (Niven and Laughlin 2008). $\mathrm{Na}^{+} /$
$\mathrm{K}^{+}$-ATPase pumps were previously suggested to be localized at the nodes of Ranvier; however, pumps are now known to also be dispersed throughout myelin internodes (McGrail and others 1991). Since humans have axons that can extend up to more than a meter in length, neural processing within these long-range, myelinated axons is metabolically expensive. Myelin sheaths also act as a barrier, restricting access to extracellular metabolites (Nave 2010). This barrier underscores the importance of oligodendrocytes in energy passage into the axon. Like axons, oligodendrocytes have high energy demands and are extremely vulnerable to energy deprivation (Pantoni and others 1996). Oligodendrocytes' particular susceptibility to energy deprivation is evidenced by the fact that occlusion of the middle cerebral artery leads to oligodendrocyte

\footnotetext{
'Department of Biology, Georgetown University, Washington, DC, USA

${ }^{2}$ Interdisciplinary Program in Neuroscience, Georgetown University, Washington, DC, USA

${ }^{3}$ Center for Cell Reprogramming, Georgetown University, Washington, DC, USA
}

\section{Corresponding Author:}

Jeffrey K. Huang, Department of Biology, Georgetown University, Regents 406, 37th and O Street, NW, Washington, DC 20057, USA. Email: jh1659@georgetown.edu 


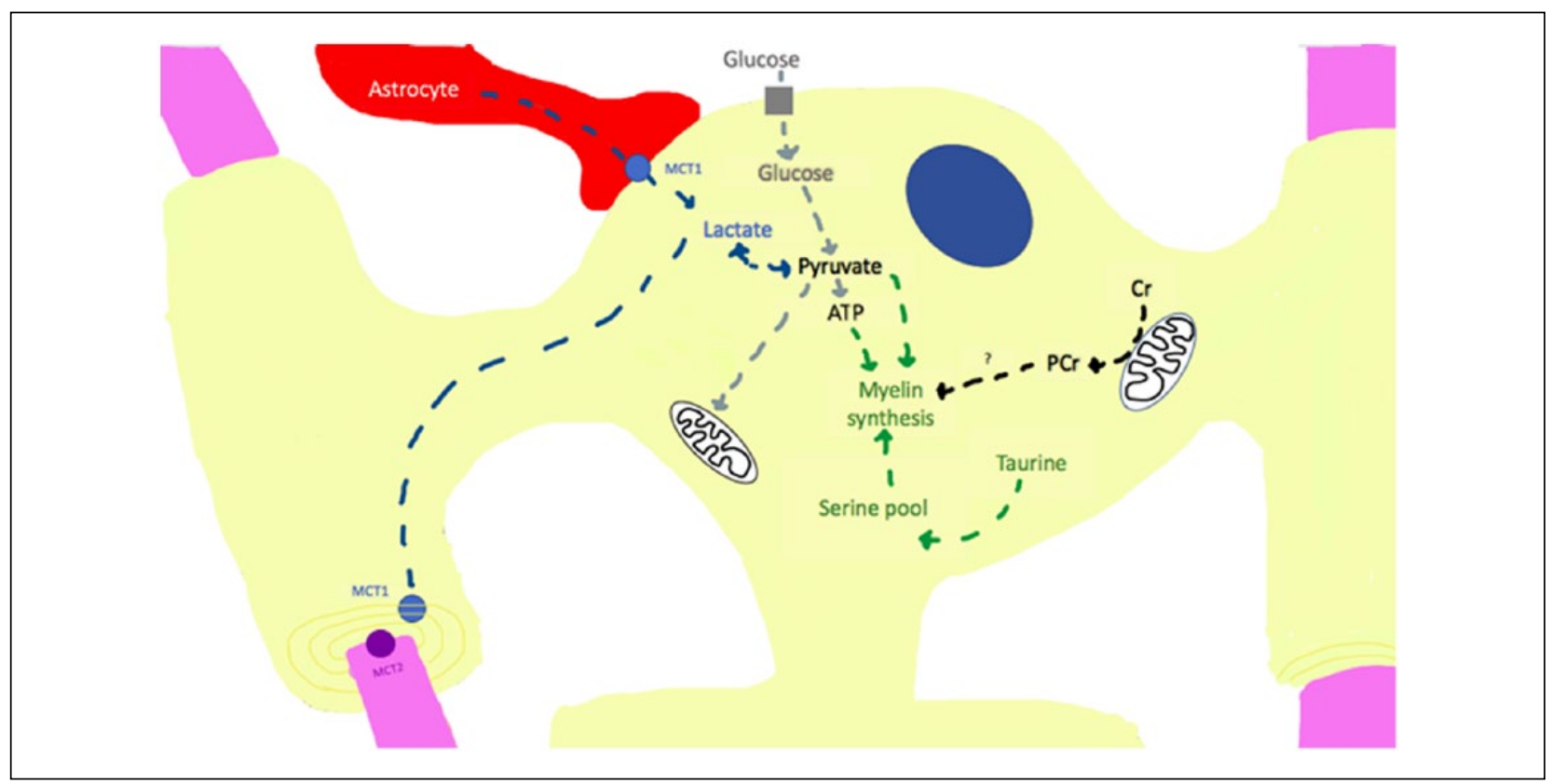

Figure I. Mechanisms of energy metabolism in the myelinating oligodendrocyte (yellow). Lactate is taken up through the monocarboxylate transporter I (MCTI) from contact with astrocytes (red). Lactate can then be shuttled to and taken up by axons (pink) through the MCT2 on neurons. Conversely, lactate can be converted to pyruvate and transported to the mitochondria for the tricarboxylic acid (TCA) cycle or converted to ATP in the cytosol by glycolysis. Glucose can also be transported into oligodendrocytes by glucose transporters and converted to pyruvate. Small metabolites, creatine and taurine, contribute to oligodendrocyte stability. Creatine $(\mathrm{Cr})$ is converted to phosphocreatine (PCr) and serves as a phosphate pool in the cytosol to aid in ATP production and promote cell survival. PCr may serve as energy source for myelin synthesis. Taurine increases serine pools to stimulate myelin synthesis within the cell.

swelling within 30 minutes and chromatin condensation within six hours (Pantoni and others 1996). These oligodendrocyte changes precede neuronal death by several hours, suggesting that energetic dysfunction in oligodendrocytes contributes to neuronal pathology. Therefore, it is important to understand how oligodendrocyte bioenergetics regulates normal brain function and how their dysfunction contributes to demyelinating diseases such as multiple sclerosis (MS).

\section{Altered Bioenergetics of Cells in MS}

MS is a chronic inflammatory demyelinating disorder of the central nervous system (CNS) (Compston and Coles 2008; Reich and others 2018). Metabolic dysfunction is commonly associated with its pathogenesis (Fig. 2) (Adiele and Adiele 2017). MS patients display acute hypoxia-like lesions with mitochondrial impairment and reduction in the expression of complex I and complex IV of the electron transport chain (Mahad and others 2008). Moreover, mitochondrial abnormalities are frequently observed in demyelinated axons and neuronal cell bodies of MS lesions (Campbell and others 2014). Impaired mitochondrial functioning can result in less available ATP in CNS lesions and trigger cell death. Dysfunctional

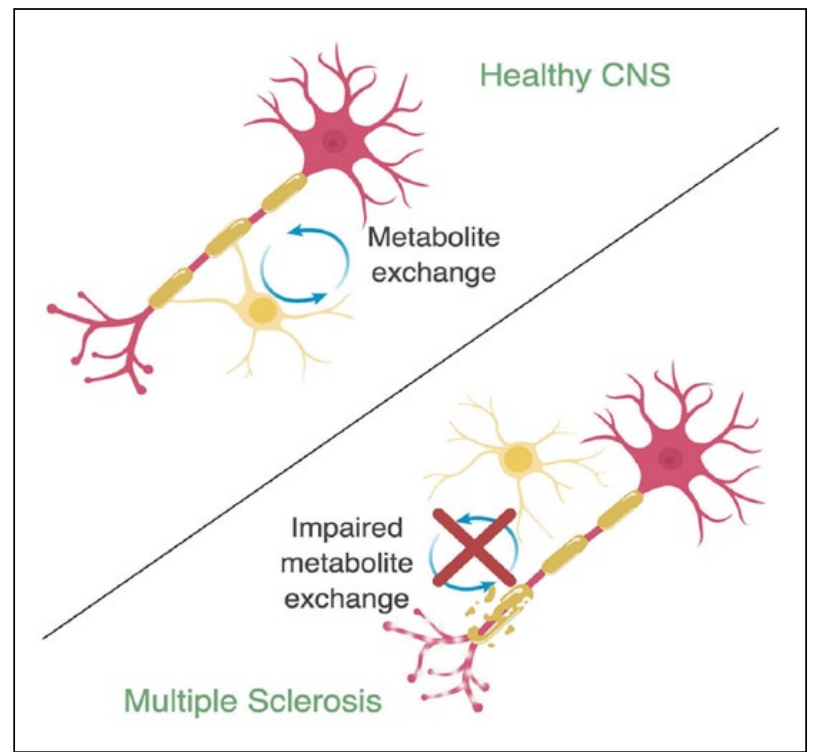

Figure 2. Metabolite exchange between oligodendrocyte (yellow) and axons (pink) in health and disease. In the healthy CNS, oligodendrocyte-axon coupling allows for bidirectional exchange of metabolites. In multiple sclerosis (MS), damage to myelin, axonal dystrophy, and dying back of oligodendrocytes leads to disrupted oligodendrocyte-axon coupling and impaired metabolite exchange. Figure created in BioRender. 
mitochondria can also lead to the increased presence of reactive oxygen species that induce peroxidation of lipids, proteins and DNA, resulting in axonal energy failure and subsequent neurodegeneration (Adiele and Adiele 2017). In addition to increased levels of reactive oxygen species, MS lesions also exhibit enhanced levels of endoplasmic reticulum (ER) stress-related proteins, CHOP, BiP, and XBP1 (Mháille and others 2008). Since mitochondria and the ER are physically associated and functionally linked to each other, chronic ER stress affects ER-mitochondria crosstalk and mitochondrial physiology, resulting in cellular pathology. The mitochondriaassociated ER membrane (MAM) plays an important role in the maintenance of cellular homeostasis by regulating calcium transfer and energy metabolism (Hayashi and others 2009). Abnormal expression of proteins associated with MAM and mitochondrial dynamics, such as Rab32, have been detected in MS lesions, suggesting that impaired MAM crosstalk may contribute to MS pathogenesis (Haile and others 2017).

Among the different patterns of demyelination in MS, one particular pattern (pattern III) is defined by oligodendrogliopathy, or the dying back of oligodendrocyte processes, which occurs prior to apoptosis (Lucchinetti and others 2000). Although the primary cause of this pattern remains poorly understood, impaired energy metabolism, either through the lack of blood supply to lesions or the local production of toxic metabolites, has been suggested to promote oligodendrogliopathy (Lassmann 2003). In animal models, the copper-chelator cuprizone, commonly used to induce demyelination and oligodendrocyte death in the corpus callosum, causes mitochondrial dysfunction in oligodendrocytes (Torkildsen and others 2008). Cuprizone decreases mitochondrial respiration by forming mitochondrial DNA dimers, leading to hypoxia-like lesions and oligodendrogliopathy in the CNS similarly to those observed in MS (Guerineau and others 1974; Lassmann 2003; Ludwin and Johnson 1981). The development of oligodendrogliopathy under cuprizone intoxication may reflect oligodendrocytes' favoring survival over myelin maintenance, similar to that which is observed in human oligodendrocytes when challenged with metabolic injury (Rone and others 2016). The presence of metabolic dysfunction in MS demands the need to better understand the role of bioenergetic metabolites and their associated mechanisms in regulating oligodendrocyte function.

\section{Glucose Fuels Oligodendrocyte Survival and Promotes Myelination}

Glucose is the main energy source for the brain (Pellerin and Magistretti 2012). During development, access to glucose is essential for myelination as well as axonal survival (Rinholm and others 2011). In fact, in vitro hypoglycemic conditions inhibit oligodendrocyte precursor cell (OPC) differentiation and migration (Yan and Rivkees 2006). Similarly, slice cultures show decreases in oligodendrocyte lineage cell numbers and myelination on glucose reduction, with severe hypoglycemia even leading to axon degeneration (Rinholm and others 2011). Intriguingly, unlike OPCs, mature oligodendrocytes rely heavily on glycolysis for ATP production even in the presence of oxygen (Funfschilling and others 2012), as shown in studies with adult rat oligodendrocytes (Rao and others 2017). Aerobic glycolysis, in which glucose is converted to lactate in the presence of oxygen, is also known as the Warburg effect, a hallmark of cancer cells (Warburg 1956). The shift to glycolysis after completion of developmental myelination suggests a strategy in which oligodendrocytes reduce mitochondria-based energetic metabolism for myelin maintenance and axonal integrity (Funfschilling and others 2012). This shift from oxidative phosphorylation to glycolysis may be necessary to reduce the production of reactive oxygen species that often occurs with active ATP synthesis.

Under normal physiological conditions, glucose is primarily metabolized aerobically into carbon dioxide and water (Zauner and others 2002). Oligodendrocyte function depends on glucose concentrations since hypoglycemia inhibits myelin development (Yan and Rivkees 2006). In fact, oxygen starvation and glucose deprivation are the two main metabolic stressors linked to neurodegeneration (Zhou and others 2018). On glucose deprivation, OPCs have fewer and thinner processes while oligodendrocytes show little change in branching morphology under stress (Zhou and others 2018), suggesting that OPCs, which have high mitochondrial demands, are highly susceptible to metabolic stress injury. Moreover, although oligodendrocytes do not display morphological changes under an acute stress condition, metabolic stress has been shown to cause oligodendrocytes to shift to a predominately glycolytic metabolism in favor of survival rather than the maintenance of myelin membranes (Rone and others 2016). In fact, lesions seen in MS patients resemble ischemic stroke injury in the CNS (Trapp and Stys 2009). Both stroke and MS lesions show degeneration of distal oligodendrocyte processes, loss of myelin, and apoptosis of oligodendrocytes (Lassmann 2016). Current research indicates that glucose availability in the brain significantly impacts the bioenergetics of oligodendrocyte lineage cells and contributes to MS and stroke pathology. Therefore, chronic metabolic stress injury, which has been suggested to occur in MS lesions, may result in remyelination failure because of OPC dysfunction and the dying back of oligodendrocyte processes over time due to the preference for oligodendrocyte survival (Rone and others 2016). Together, these findings 
suggest that strategies to enhance the glycolytic pathway in oligodendrocyte lineage cells would improve remyelination, and at the same time, ensure the survival of oligodendrocytes and axons.

\section{Lactate Promotes Oligodendrocyte- Axon Coupling}

Under a low glucose environment, lactate may support oligodendrocyte development and myelination. Lactate has been shown to promote cell cycling and differentiation of OPCs in vitro (Ichihara and others 2017). Moreover, addition of lactate to brain slices cultured in low glucose conditions was shown to rescue oligodendrocyte lineage cells and promote remyelination (Rinholm and others 2011). Interestingly, oligodendrocytes may actually prefer lactate over glucose as a substrate for myelin production, as lactate application to brain slices resulted in greater myelination than in slices treated with glucose (Rinholm and others 2011). Mature oligodendrocytes express monocarboxylate transporter 1 (MCT1), a selective transporter for lactate, to supply neurons with lactate during high neuronal activity. MCT1 is necessary for maintaining axonal integrity, as mice with reduced MCT1 expression exhibit axonal swelling and distended mitochondria by 8 months of age (Lee and others 2012). Furthermore, reduction of MCT1 in oligodendrocytes is sufficient to induce axonal dystrophy, suggesting that lactate supply from oligodendrocytes is essential for axonal health (Funfschilling and others 2012). Therefore, lactate is critical for oligodendrocyte function and oligodendrocyte-axon coupling.

Several studies have shown that disruption of oligodendrocyte-axon coupling may contribute to disease pathogenesis. After ischemic injury in the CNS, striatum levels of MCT1 are significantly upregulated in animals following reperfusion of the middle cerebral artery (Zhou and others 2018). Oxygen-glucose deprivation from ischemia triggers the upregulation of MCT1 in OPCs to allow increased transport of lactate from astrocytes and from the blood. However, prolonged periods of stress can result in the failure of oligodendrocytes to produce sufficient energy for the maintenance of axons, thus leading to axonal degradation (Zhou and others 2018). In amyotrophic lateral sclerosis (ALS) patients, and in the ALS mouse model, SOD1 ${ }^{\mathrm{G} 93 \mathrm{~A}}$ mutant, MCT1 expression in the CNS is significantly reduced (Lee and others, 2012). Since lactate supply from oligodendrocytes is critical for axonal function and neuronal survival, alterations of MCT1 expression in oligodendrocytes may contribute to ALS pathogenesis. In MS, acute lesions have been shown to be hypermetabolic (Schiepers and others 1997), suggesting prolonged periods of metabolic stress can lead to OPC dysfunction and oligodendrocytes' failure to provide lactate to axons. Therefore, improving oligodendrocyte bioenergetics would enhance oligodendrocyte-axon coupling and promote neuroprotection in neurodegenerative diseases.

\section{Taurine Preserves Mitochondrial Function and Promotes Oligodendrocyte Differentiation}

Taurine has recently been shown to have an important role in oligodendrocyte function (Beyer and others 2018). Taurine is a sulfurous, semi-essential amino acid found throughout animal tissues which can be synthesized either by the conversion of methionine and cysteine or taken in by diet (De la Puerta and others 2010). Taurine is primarily synthesized in the liver, then exported, and taken up by organs through a sodium and chloride dependent taurine transporter (Pasantes-Morales and Hernández-Benítez 2010). It is thought to have many biological roles, including aiding in mitochondrial $\mathrm{pH}$ buffering capacity and preventing mitochondria swelling and damage (Hansen and others 2010). It also stabilizes oxidative phosphorylation in muscle and heart mitochondria (Scholte and others 1997) and exerts neuroprotective effects during oxidative stress (Xu and others 2015). Moreover, taurine decreases the expression of ER stressrelated proteins and prevents ER stress-induced apoptosis in mammalian cells by increasing the antiapoptotic molecule Bcl-2 and reducing the expression of apoptosisinducing proteins cytochrome $\mathrm{c}$ and cleaved caspase 3 (Zhang and others 2016; Zhang and others 2017). In addition to promoting cell survival, taurine impacts oligodendrocyte function by enhancing differentiation. Recent studies show that taurine levels are significantly elevated during oligodendrocyte differentiation (Beyer and others 2018). Moreover, exogenous addition of taurine to OPC cultures enhances oligodendrocyte differentiation and maturation (Beyer and others 2018), suggesting that taurine may be synthesized in oligodendrocyte lineage cells to stimulate differentiation (Fig. 3). It was also found that taurine directly increases the availability of intracellular serine, an amino acid that serves as a critical building block for glycosphingolipid synthesis in myelination (Beyer and others 2018). The taurine-driven upregulation of serine in oligodendrocytes indicates that taurine may be necessary for initiating lipid biogenesis during myelination. Thus, taurine supplementation may improve remyelination in MS by promoting oligodendrocyte differentiation and maturation, in additional to enhancing mitochondrial function, reducing oxidative and ER stress, and preventing metabolic stress-related apoptosis. 


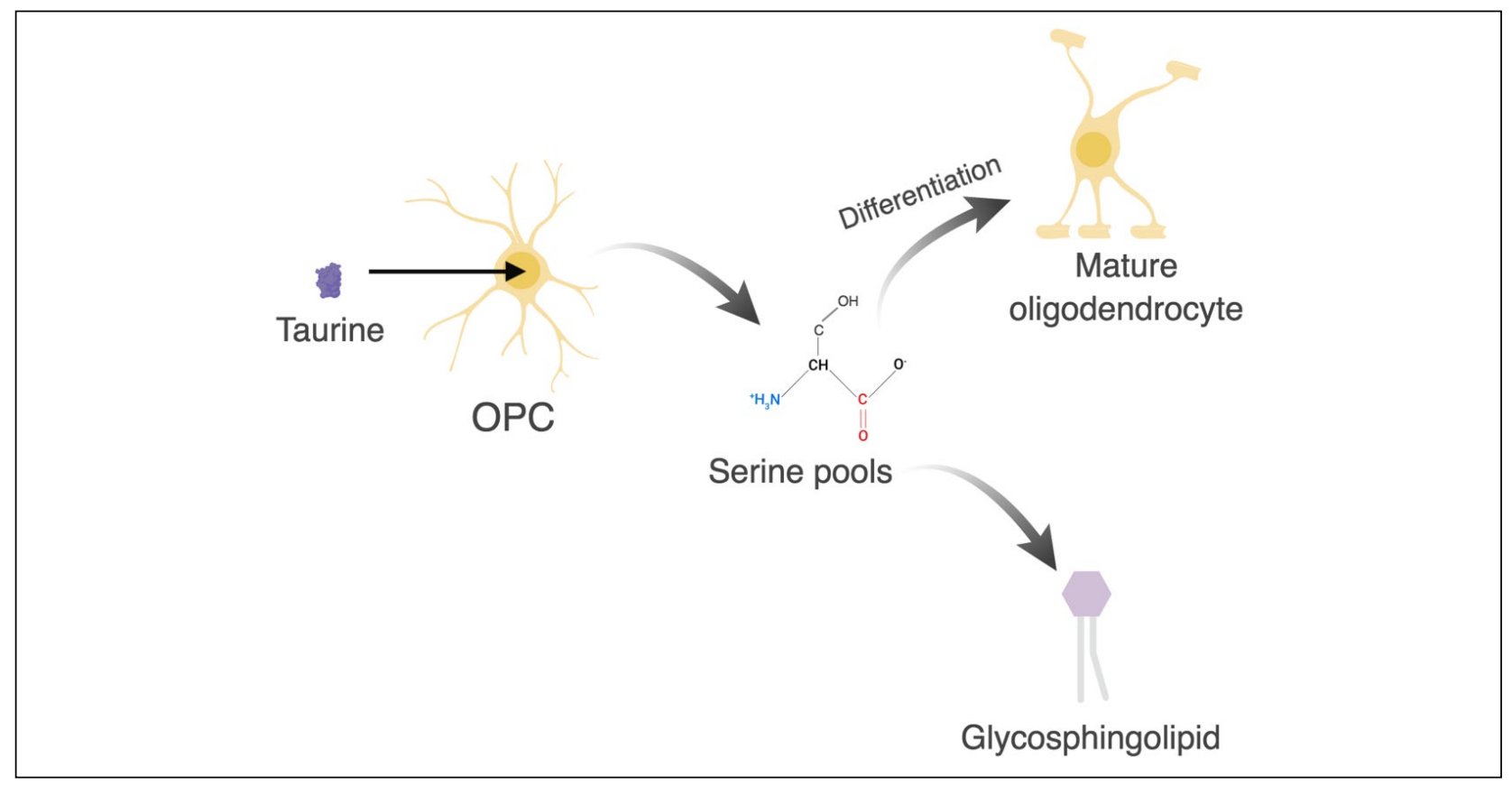

Figure 3. Uptake of taurine in oligodendrocyte precursor cells (OPCs) promotes differentiation and myelin synthesis. Taurine increases serine pools which enhances oligodendrocyte differentiation and promotes glycosphingolipid synthesis. Glycosphingolipid biosynthesis is essential for myelin synthesis. Figure created in BioRender.

\section{Creatine Promotes Oligodendrocyte Survival by Improving Mitochondria Function}

Creatine, a nitrogenous organic acid, is a semi-essential metabolite important for healthy cell function. Fifty percent of creatine is synthesized within the body by the conversion of arginine and glycine and the other $50 \%$ is taken up from diet (Brosnan and Brosnan 2007). It is distributed throughout the body through the bloodstream and taken up by cells using the transporter SLC6A8 (Wyss and Kaddurah-Daouk 2000). Creatine plays a critical role in ATP buffering in times of high energy demands and, accordingly, is found in tissues with largely fluctuating energy demands, such as brain and muscle (Wyss and Kaddurah-Daouk 2000). Exogenously supplied creatine has been shown to be neuroprotective under hypoxic conditions by reducing the need for oxygen to replenish ATP. Creatine has been shown to enhance mitochondrial membrane potential and increase synaptic density in hippocampal neurons ( $\mathrm{Li}$ and others 2004). Similarly, treating muscle fibers with creatine improves mitochondrial function by increasing ADP-stimulated respiration (Walsh and others 2001). In addition to the effect of creatine on hippocampal neurons and muscle fibers, exogenously applied creatine has been shown to significantly increase mitochondrial density, membrane potential, and ATP production in oligodendrocytes (Fig. 4) (Chamberlain and others 2017). Creatine administration was found to improve mitochondrial function and survival in oligodendrocytes without affecting membrane expansion or oligodendrocyte differentiation (Chamberlain and others 2017). Thus, creatine has a positive effect on the bioenergetics of oligodendrocytes and dietary supplementation may provide a therapeutic benefit to MS patients.

Although creatine from diet is able to cross the blood brain barrier, it does so with poor efficiency (Béard and Braissant 2010). The lack of creatine transporter expression in astrocytes suggests that endogenous creatine synthesis may take place within the CNS, with transport among CNS cells, rather than through uptake of creatine circulating in the blood (Braissant and Henry 2008; Braissant 2012). Biosynthesis of endogenous creatine in the CNS involves the enzyme L-arginine:glycine amidinotransferase (AGAT), which converts arginine and glycine into guanidinoacetate. The formation of guanidinoacetate is then followed by its conversion to creatine by the enzyme guanidinoacetate methyltransferase (GAMT). The ability to synthesize creatine in the brain is crucial for CNS function, given creatine's role in oligodendrocytes. In the CNS, AGAT is expressed in all glial cells, whereas GAMT is primarily expressed in oligodendrocytes, and SLC6A8, the creatine transporter, is found in both oligodendrocytes and neurons (Braissant and others 2001; Chamberlain and others 2017). Since oligodendrocytes are the main producers of endogenous 

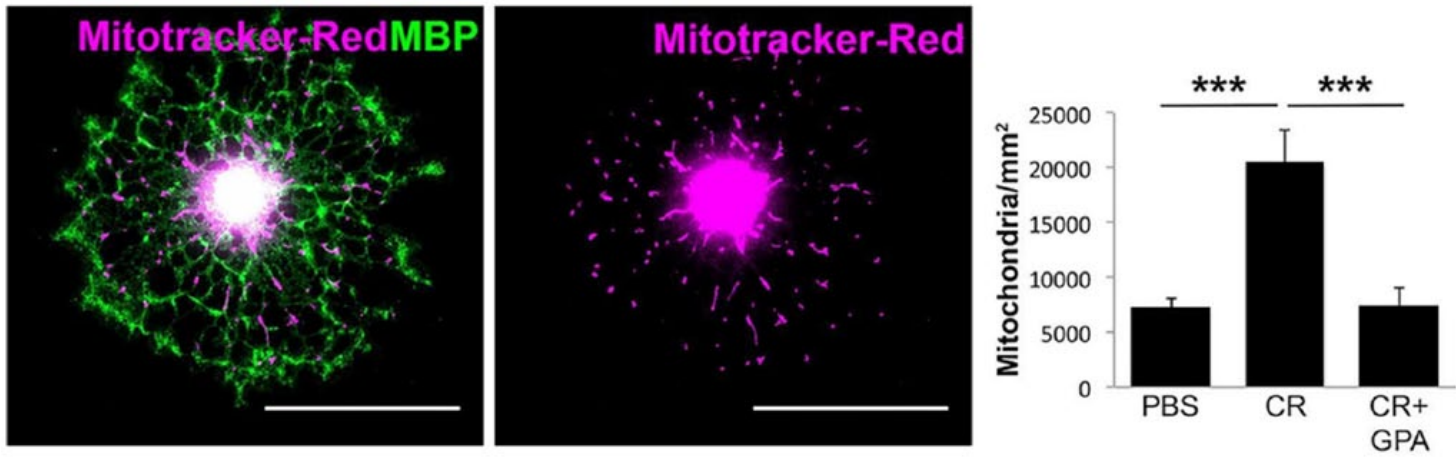

Figure 4. Creatine increases mitochondrial density in oligodendrocytes. A significant increase in mitochondria density was calculated in myelin basic protein (MBP)-positive oligodendrocytes after treatment with $100 \mu \mathrm{M}$ creatine (CR) for 24 hours. Oligodendrocytes treated with PBS or treated with creatine plus guanidinopropionic acid (GPA), a competitive antagonist to the creatine transporter, showed similar mitochondrial density. Modified from Chamberlain and others (2017).

creatine, their function might rely heavily on endogenously synthesized creatine, or they might play a role in shuttling creatine to other cells in the CNS. Individuals with creatine synthesis-related mutations, such as those in AGAT, GAMT, or SLC6A8, collectively referred to as inborn errors of metabolism in the creatine synthesis pathway, display profound intellectual disability, seizures, and impaired myelination (Braissant and others 2010). Despite the well-known importance of creatine in the body, the role of creatine in brain cells remains poorly understood.

Mice lacking Gamt expression and raised under creatine deficient diet display reduced body weight and metabolic alterations compared to wildtype mice, but otherwise are viable with no gross behavioral abnormalities (Schmidt and others 2004). Under regular diet, Gamt-deficient mice display normal body weight and no obvious CNS abnormalities, suggesting that creatine derived from diet may be sufficient to compensate for the lack of endogenously synthesized creatine (Chamberlain and others 2017). However, following experimental demyelination, Gamtdeficient mice fed with regular diet display significantly fewer mature oligodendrocytes in lesions compared to wildtype animals. Interestingly, OPCs do not appear to be affected in these mice. Instead, significant apoptosis of newly generated oligodendrocytes was observed in these lesions. Moreover, creatine administration to demyelinated CNS lesions rescued oligodendrocyte density and improved remyelination in Gamt-deficient mice. These results suggest that creatine synthesis is required for the survival of newly generated oligodendrocytes during remyelination and that the administration of creatine into the CNS significantly improves oligodendrocyte survival in lesions after demyelinating injury (Chamberlain and others 2017).
Creatine supplementation has been considered for the treatment of many neurodegenerative diseases such as Alzheimer's disease, Parkinson's disease, Huntington's disease and ALS - all of which exhibit mitochondrial dysfunction (Adhihetty and Beal 2008). Increasing creatine levels in the CNS may be particularly helpful for MS patients, since mitochondrial dysfunction and oligodendrocyte death are key events in disease pathology. However, the limited diffusion of peripherally derived creatine across the blood brain barrier in the CNS remains an issue that must be overcome if creatine supplementation were considered for the treatment of neurological disorders. The use of cyclocreatine, a planar creatine ana$\log$ that crosses the blood brain barrier more readily (Kurosawa and others 2012), may be a more suitable therapy for improving bioenergetics and protecting CNS cells in neurological disorders.

\section{N-Acetylaspartate and Biotin Also Regulate Myelin Synthesis and Energy Production}

Several other metabolites such as $N$-acetylaspartate (NAA) and biotin are known to affect oligodendrocyte function and promote myelin synthesis. NAA is a neuronal mitochondrial metabolite that likely promotes crosstalk between neurons and oligodendrocytes, as neurons contain aspartate $N$-acetyltransferase, the enzyme required for NAA synthesis, and oligodendrocytes contain aspartoacylase (ASPA), the enzyme necessary for NAA metabolism into acetate and aspartate ( $\mathrm{Li}$ and others 2013; Moffett and others 2011). NAA is used by oligodendrocytes for energy production rather than being released back to neurons for NAA recycling and use, as was previously hypothesized (Amaral and others 2017). 




Figure 5. Neuronal-derived $\mathrm{N}$-acetylacetate (NAA) promotes myelin synthesis and energy production in oligodendrocytes (yellow). NAA is synthesized in neurons (pink) from acetyl-CoA and aspartate by aspartate $N$-acetyltransferase (Asp-NAT). In oligodendrocytes, NAA is metabolized by aspartoacylase (ASPA) into acetate and aspartate, which are used for myelin synthesis and energy production via the malate-aspartate shuttle (Mal-Asp shuttle), respectively. Figure created in BioRender.

NAA-derived aspartate is likely used in the malate-aspartate shuttle to transport reducing equivalents into the mitochondria for oxidative phosphorylation and ATP production (Amaral and others 2017). NAA also provides oligodendrocytes with the acetate residues necessary for lipogenesis and myelin synthesis (Fig. 5) (Hagenfeldt and others 1987). Thus, NAA is necessary for the synthesis and function of healthy myelin. In fact, impaired NAA metabolism is implicated in several neurodegenerative disorders impacting myelin, including Canavan disease and MS. Canavan disease is a neurodegenerative leukodystrophy caused by mutations in the gene encoding ASPA (Hoshino and Kubota 2014). In Canavan disease, ASPA deficiency prevents NAA breakdown into acetate and aspartate, resulting in reduced myelin synthesis and spongy white matter degeneration (Hoshino and Kubota 2014). Reduced NAA-derived acetate levels are also implicated in multiple sclerosis. In the brains of people with MS, low NAA levels are correlated with decreased availability of acetate in white matter ( $\mathrm{Li}$ and others 2013). Furthermore, decreased NAA levels are correlated with clinical disability and cognitive impairment, as well as axonal loss and brain atrophy, in MS (Li and others 2013).

Similar to NAA, biotin promotes myelin synthesis and energy production. Biotin, also known as vitamin $\mathrm{H}$, is a B-complex vitamin which acts as a coenzyme for five carboxylases involved in fatty acid synthesis and energy production (Zempleni and others 2009). Biotin is essential for brain health, as many patients with deficiencies in biotin recycling have neurological dysfunction with symptoms, including seizures, cognitive deficits, and hypotonia (Wolf 2011). Recent studies suggest that biotin is important for the health and function of both oligodendrocytes and neurons. In oligodendrocytes, biotin promotes myelin synthesis through binding with acetyl-CoA carboxylase (ACC1 and ACC2) and increasing the availability of fatty acids (Sedel and others 2016). In neurons, biotin reduces hypoxia by binding with enzymes necessary for the formation of TCA cycle intermediates and increasing ATP production (Fig. 6) (Sedel and others, 2016). Biotin supplementation, then, could potentially promote myelin repair and reduce hypoxia-induced axonal degeneration in the brains of people with MS, 


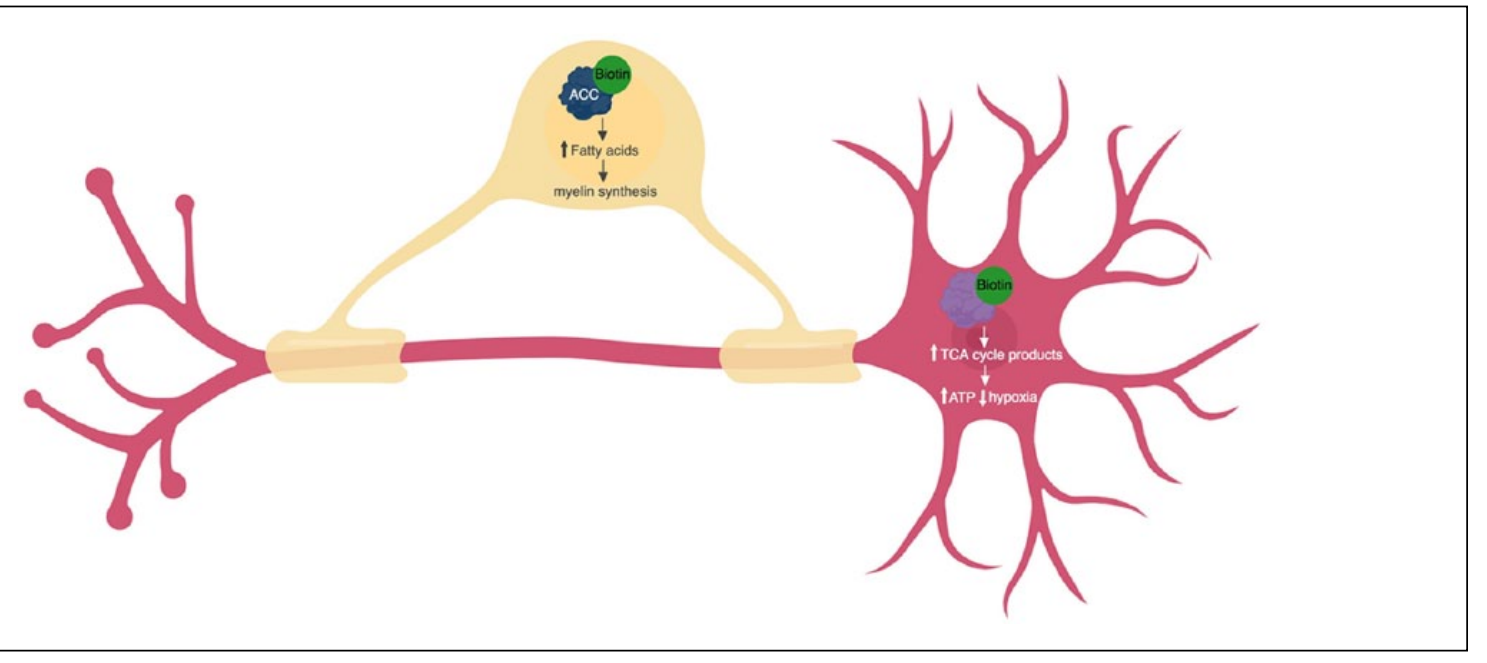

Figure 6. Biotin promotes myelin synthesis and energy production by acting as a coenzyme in oligodendrocytes (yellow) and neurons (pink). In oligodendrocytes, biotin (green) binds with acetyl-CoA carboxylase (ACCI and ACC2) (blue) and increases the availability of fatty acids, allowing increased myelin synthesis. In neurons, biotin binds tricarboxylic acid (TCA) cycle enzymes (pyruvate carboxylase, 3-methylcrotonyl-CoA carboxylase, propionyl-CoA carboxylase) (purple) and increases production of TCA cycle intermediates, increasing ATP production and reducing neuronal hypoxia. Figure created in BioRender.

particularly important for those with progressive MS who have spontaneous remyelination failure and significant axonal degeneration. In a recent pilot study, researchers found that high-dose biotin supplementation $(100-600$ $\mathrm{mg} /$ day) improved the clinical symptoms of $89 \%$ of progressive MS patients with sensory and motor impairments (Sedel and others 2015). Importantly, the delayed onset (2-8 months) of patients' clinical improvement is consistent with biotin-mediated myelin remodeling (Sedel and others 2015; Sedel and others 2016). In a larger, double-blind, placebo-controlled study, high-dose biotin supplementation (300 $\mathrm{mg} /$ day) reduced the MS-related disability and disease progression of $12.6 \%$ of progressive MS patients, compared with no improvement in the placebo group (Tourbah and others 2016). Of the $12.6 \%$ of patients with improved MS symptoms, the majority of them (77\%) had sustained improvement after 2 years of biotin treatment (Tourbah and others 2016). Both NAA and biotin are essential for bioenergetics and myelin synthesis, suggesting that therapies which maintain healthy levels of these metabolites could potentially improve the symptoms of those with MS and other neurodegenerative disorders.

\section{Conclusion}

Coupling of axons and oligodendrocytes is essential for healthy neuronal signaling. In addition to generating myelin sheaths to increase the speed of neuronal firing, oligodendrocytes supply neurons with metabolites to maintain neuronal integrity and promote survival. During development, OPCs require oxygen to function properly, but once differentiated, oligodendrocytes shift into primarily glycolytic metabolism. During pathological CNS states, there are fluctuating periods of glucose and oxygen deprivation, leading to changes in bioenergetics of oligodendrocytes. However, severe metabolic stresses prevent oligodendrocytes from supporting axonal integrity. Understanding the bioenergetic mechanisms of oligodendrocytes is essential to explain the dysfunction of oligodendrocyte lineage cells in MS. Here, we described the improvement of mitochondrial function using taurine and creatine supplementation. We also discussed the importance of NAA and biotin in myelin synthesis and energy production. We suggest that disturbances in oligodendrocyte bioenergetics may be an early event in MS pathology and that increasing bioenergetic metabolites in the CNS through supplementation may aid in improving mitochondrial function and oligodendrocyte survival in MS.

\section{Declaration of Conflicting Interests}

The author(s) declared no potential conflicts of interest with respect to the research, authorship, and/or publication of this article.

\section{Funding}

The author(s) disclosed receipt of the following financial support for the research, authorship, and/or publication of this article: This work was supported by grants from National Multiple Sclerosis Society (PP160624509) and Congressionally Directed Medical Research Programs (W81XWH-17-1-0268) to J.K.H. 


\section{ORCID iD}

Jeffrey K. Huang (iD https://orcid.org/0000-0003-0996-9451

\section{References}

Adhihetty PJ, Beal MF. (2008). Creatine and its potential therapeutic value for targeting cellular energy impairment in neurodegenerative diseases. Neuromolecular Med 10:27590.

Adiele RC, Adiele CA. (2017). Metabolic defects in multiple sclerosis. Mitochondrion. Epub Dec 13. doi:10.1016/j. mito.2017.12.005.

Amaral AI, Hadera MG, Kotter M, Sonnewald U. (2017). Oligodendrocytes do not export NAA-derived aspartate in vitro. Neurochem Res 42:827-37.

Béard E, Braissant O. (2010). Synthesis and transport of creatine in the CNS: importance for cerebral functions. J Neurochem 115:297-313.

Beyer BA, Fang M, Sadrian B, Montenegro-Burke JR, Plaisted WC, Kok BPC, and others. (2018). Metabolomics-based discovery of a metabolite that enhances oligodendrocyte maturation. Nat Chem Biol 14:22-8.

Bradl M, Lassmann H. (2010). Oligodendrocytes: biology and pathology. Acta Neuropathol 119:37-53.

Braissant O. (2012). Creatine and guanidinoacetate transport at blood-brain and blood-cerebrospinal fluid barriers. J Inherit Metab Dis 35:655-64.

Braissant O, Béard E, Torrent C, Henry H. (2010). Dissociation of AGAT, GAMT and SLC6A8 in CNS: relevance to creatine deficiency syndromes. Neurobiol Dis 37:423-33.

Braissant O, Henry H. (2008). AGAT, GAMT and SLC6A8 distribution in the central nervous system, in relation to creatine deficiency syndromes: a review. J Inherit Metab Dis 31:230-9.

Braissant O, Henry H, Loup M, Eilers B, Bachmann C. (2001). Endogenous synthesis and transport of creatine in the rat brain: an in situ hybridization study. Brain Res Mol Brain Res 86:193-201.

Brosnan JT, Brosnan ME. (2007). Creatine: endogenous metabolite, dietary, and therapeutic supplement. Annu Rev Nutr 27:241-61.

Campbell GR, Worrall JT, Mahad DJ. (2014). The central role of mitochondria in axonal degeneration in multiple sclerosis. Mult Scler 20:1806-13.

Chamberlain KA, Chapey KS, Nanescu SE, Huang JK. (2017). Creatine enhances mitochondrial-mediated oligodendrocyte survival after demyelinating injury. J Neurosci 37:1479-92.

Compston A, Coles A. (2008). Multiple sclerosis. Lancet 372:1502-17.

De la Puerta C, Arrieta FJ, Balsa JA, Botella-Carretero JI, Zamarrón I, Vázquez C. (2010). Taurine and glucose metabolism: a review. Nutr Hosp 25:910-9.

Funfschilling U, Supplie LM, Mahad D, Boretius S, Saab AS, Edgar J, and others. (2012). Glycolytic oligodendrocytes maintain myelin and long-term axonal integrity. Nature 485:517-21.
Griffiths I, Klugmann M, Anderson T, Yool D, Thomson C, Schwab MH, and others. (1998). Axonal swellings and degeneration in mice lacking the major proteolipid of myelin. Science 280:1610-3.

Guerineau M, Guerineau S, Gosse C. (1974). Abnormal mitochondrial DNA molecules in megamitochondria from cuprizone-treated rats. Eur J Biochem 47:313-9.

Hagenfeldt L, Bollgren I, Venizelos N. (1987). $N$-acetylaspartic aciduria due to aspartoacylase deficiency - a new aetiology of childhood leukodystrophy. J Inherit Metab Dis 10:13541.

Haile Y, Deng X, Ortiz-Sandoval C, Tahbaz N, Janowicz A, Lu J-Q, and others. (2017). Rab32 connects ER stress to mitochondrial defects in multiple sclerosis. J Neuroinflammation 14:19.

Hansen SH, Andersen ML, Cornett C, Gradinaru R, Grunnet N. (2010). A role for taurine in mitochondrial function. J Biomed Sci 17(Suppl 1):S23.

Harris JJ, Attwell D. (2012). The energetics of CNS white matter. J Neurosci 32:356-71.

Hayashi T, Rizzuto R, Hajnoczky G, Su T-P. (2009). MAM: more than just a housekeeper. Trends Cell Biol 19:81-8.

Hoshino H, Kubota M. (2014). Canavan disease: clinical features and recent advances in research. Pediatr Int 56:47783.

Ichihara Y, Doi T, Ryu Y, Nagao M, Sawada Y, Ogata T. (2017). Oligodendrocyte progenitor cells directly utilize lactate for promoting cell cycling and differentiation. J Cell Physiol 232:986-95.

Kurosawa Y, Degrauw TJ, Lindquist DM, Blanco VM, PyneGeithman GJ, Daikoku T, and others. (2012). Cyclocreatine treatment improves cognition in mice with creatine transporter deficiency. J Clin Invest 122:2837-46.

Lassmann H. (2003). Hypoxia-like tissue injury as a component of multiple sclerosis lesions. J Neurol Sci 206:187-91.

Lassmann H. (2016). Demyelination and neurodegeneration in multiple sclerosis: the role of hypoxia. Ann Neurol 79:520-1.

Lee Y, Morrison BM, Li Y, Lengacher S, Farah MH, Hoffman PN, and others. (2012). Oligodendroglia metabolically support axons and contribute to neurodegeneration. Nature 487:443-8.

Li S, Clements R, Sulak M, Gregory R, Freeman E, McDonough J. (2013). Decreased NAA in gray matter is correlated with decreased availability of acetate in white matter in postmortem multiple sclerosis cortex. Neurochem Res 38:2385-96.

Li Z, Okamoto K-I, Hayashi Y, Sheng M. (2004). The importance of dendritic mitochondria in the morphogenesis and plasticity of spines and synapses. Cell 119:873-87.

Lucchinetti C, Brück W, Parisi J, Scheithauer B, Rodriguez M, Lassmann H. (2000). Heterogeneity of multiple sclerosis lesions: implications for the pathogenesis of demyelination. Ann Neurol 47:707-17.

Ludwin SK, Johnson ES. (1981). Evidence for a "dying-back" gliopathy in demyelinating disease. Ann Neurol 9:301-5.

Mahad D, Ziabreva I, Lassmann H, Turnbull D. (2008). Mitochondrial defects in acute multiple sclerosis lesions. Brain 131:1722-35. 
McGrail KM, Phillips JM, Sweadner KJ. (1991). Immunofluorescent localization of three $\mathrm{Na}, \mathrm{K}$-ATPase isozymes in the rat central nervous system: both neurons and glia can express more than one Na,K-ATPase. J Neurosci 11:381-91.

McLaurin JA, Yong VW. (1995). Oligodendrocytes and myelin. Neurol Clin 13:23-49.

Mháille AN, McQuaid S, Windebank A, Cunnea P, McMahon J, Samali A, and others. (2008). Increased expression of endoplasmic reticulum stress-related signaling pathway molecules in multiple sclerosis lesions. J Neuropathol Exp Neurol 67:200-11.

Moffett JR, Arun P, Ariyannur PS, Garbern JY, Jacobowitz DM, Namboodiri AMA. (2011). Extensive aspartoacylase expression in the rat central nervous system. Glia 59:141434.

Nave K-A. (2010). Myelination and the trophic support of long axons. Nat Rev Neurosci 11:275-83.

Niven JE, Laughlin SB. (2008). Energy limitation as a selective pressure on the evolution of sensory systems. J Exp Biol 211:1792-804.

Pantoni L, Garcia JH, Gutierrez JA. (1996). Cerebral white matter is highly vulnerable to ischemia. Stroke 27:1641-6.

Pasantes-Morales H, Hernández-Benítez R. (2010). Taurine and brain development: trophic or cytoprotective actions? Neurochem Res 35:1939-43.

Pellerin L, Magistretti PJ. (2012). Sweet sixteen for ANLS. J Cereb Blood Flow Metab 32:1152-66.

Rao VTS, Khan D, Cui Q-L, Fuh S-C, Hossain S, Almazan $\mathrm{G}$, and others. (2017). Distinct age and differentiationstate dependent metabolic profiles of oligodendrocytes under optimal and stress conditions. PLoS ONE 12: e0182372.

Reich DS, Lucchinetti CF, Calabresi PA. (2018). Multiple sclerosis. N Engl J Med 378:169-80.

Rinholm JE, Hamilton NB, Kessaris N, Richardson WD, Bergersen LH, Attwell D. (2011). Regulation of oligodendrocyte development and myelination by glucose and lactate. J Neurosci 31:538-48.

Rone MB, Cui Q-L, Fang J, Wang L-C, Zhang J, Khan D, and others. (2016). Oligodendrogliopathy in multiple sclerosis: low glycolytic metabolic rate promotes oligodendrocyte survival. J Neurosci 36:4698-707.

Schiepers C, Van Hecke P, Vandenberghe R, Van Oostende S, Dupont P, Demaerel P, and others. (1997). Positron emission tomography, magnetic resonance imaging and proton NMR spectroscopy of white matter in multiple sclerosis. Mult Scler 3:8-17.

Schmidt A, Marescau B, Boehm EA, Renema WK, Peco R, Das A, and others. (2004). Severely altered guanidino compound levels, disturbed body weight homeostasis and impaired fertility in a mouse model of guanidinoacetate $\mathrm{N}$-methyltransferase (GAMT) deficiency. Hum Mol Genet 13:905-21.

Scholte HR, Yu Y, Ross JD, Oosterkamp II, Boonman AM, Busch HF. (1997). Rapid isolation of muscle and heart mitochondria, the lability of oxidative phosphorylation and attempts to stabilize the process in vitro by taurine, carnitine and other compounds. Mol Cell Biochem 174:61-6.

Sedel F, Bernard D, Mock DM, Tourbah A. (2016). Targeting demyelination and virtual hypoxia with high-dose biotin as a treatment for progressive multiple sclerosis. Neuropharmacology 110:644-53.

Sedel F, Papeix C, Bellanger A, Touitou V, Lebrun-Frenay C, Galanaud D, and others. (2015). High doses of biotin in chronic progressive multiple sclerosis: a pilot study. Mult Scler Relat Disord 4:159-69.

Torkildsen O, Brunborg LA, Myhr K-M, Bø L. (2008). The cuprizone model for demyelination. Acta Neurol Scand Suppl 188:72-6.

Tourbah A, Lebrun-Frenay C, Edan G, Clanet M, Papeix C, Vukusic S, and others. (2016). MD1003 (high-dose biotin) for the treatment of progressive multiple sclerosis: a randomised, double-blind, placebo-controlled study. Mult Scler 22:1719-31.

Trapp BD, Stys PK. (2009). Virtual hypoxia and chronic necrosis of demyelinated axons in multiple sclerosis. Lancet Neurol 8:280-91.

Walsh B, Tonkonogi M, Söderlund K., Hultman E, Saks V, Sahlin K. (2001). The role of phosphorylcreatine and creatine in the regulation of mitochondrial respiration in human skeletal muscle. J Physiol 537:971-8.

Warburg O. (1956). On the origin of cancer cells. Science 123:309-14.

Wolf B. (2011). The neurology of biotinidase deficiency. Mol Genet Metab 104:27-34.

Wyss M, Kaddurah-Daouk R. (2000). Creatine and creatinine metabolism. Physiol Rev 80:1107-213.

Xu S, He M, Zhong M, Li L, Lu Y, Zhang Y, and others. (2015). The neuroprotective effects of taurine against nickel by reducing oxidative stress and maintaining mitochondrial function in cortical neurons. Neurosci Lett 590:52-7.

Yan H, Rivkees SA. (2006). Hypoglycemia influences oligodendrocyte development and myelin formation. Neuroreport 17:55-9.

Zauner A, Daugherty WP, Bullock MR, Warner DS. (2002). Brain oxygenation and energy metabolism: part I-biological function and pathophysiology. Neurosurgery 51:289301; discussion 302.

Zempleni J, Wijeratne SSK, Hassan YI. (2009). Biotin Biofactors 35:36-46.

Zhang Y, Li D, Li H, Hou D, Hou J. (2016). Taurine pretreatment prevents isoflurane-induced cognitive impairment by inhibiting ER stress-mediated activation of apoptosis pathways in the hippocampus in aged rats. Neurochem Res 41:2517-25.

Zhang Y, Ren S, Liu Y, Gao K, Liu Z, Zhang Z. (2017). Inhibition of starvation-triggered endoplasmic reticulum stress, autophagy, and apoptosis in ARPE-19 cells by taurine through modulating the expression of calpain-1 and calpain-2. Int J Mol Sci 18:2146.

Zhou P, Guan T, Jiang Z, Namaka M, Huang Q-J, Kong J-M. (2018). Monocarboxylate transporter 1 and the vulnerability of oligodendrocyte lineage cells to metabolic stresses. CNS Neurosci Ther 24:126-34. 